\title{
Disparidades por etnia en la mortalidad por minas antipersona: un análisis a nivel país
}

\author{
Ethnic disparities in mortality from landmines: A country-level analysis
}

José G. Rodríguez-Narváez¹, Andrea Castillo¹, Jessica González¹, Alejandro González¹, Ramiro Manzano-Núñez ${ }^{1,2}$ (D) Felipe González¹, Alberto F. García ${ }^{3}$ (D)

1 Médico, residente de Cirugía general, Universidad del Rosario, MÉDERI Hospital Universitario Mayor, Bogotá, D.C., Colombia.

2 Barco Hospital San Raffaele, Cali, Colombia.

3 Médico, especialista en Cirugía general y Cirugía de Trauma y Emergencias; División de Cirugía de Trauma y Emergencias, departamento de Cirugía General, Fundación Valle del Lili; profesor, departamento de Cirugía General, Hospital Universitario del Valle Evaristo García, Universidad del Valle, Cali, Colombia.

\section{Resumen}

Introducción. El Registro Colombiano de Víctimas de Lesiones por Minas Antipersona fue lanzado por el gobierno de Colombia con el objetivo de recolectar información sobre los casos de heridos por minas antipersona en el país. El propósito de este estudio fue investigar las disparidades de mortalidad entre las víctimas de lesiones por minas antipersona, en función de la pertenencia a una minoría étnica.

Métodos. Se hizo una regresión logística multivariable para examinar la asociación entre minorías étnicas y mortalidad en las personas heridas por minas antipersona.

Resultados. Se registraron 10.306 casos de lesiones por minas antipersona, de los cuales 430 eran personas pertenecientes a grupos étnicos minoritarios (indígenas o afrodescendientes). De estos, 85 (19,7 \%) eran mujeres, 156 (36,2 \%) eran menores de 18 años y 427 (99,3\%) vivían en áreas rurales. La mortalidad fue significativamente mayor $(29,3 \%)$ en comparación con la población mestiza $(18,5 \%$; p < 0,001). Después de ajustar por sexo, edad, soldado en servicio activo, área rural y volumen de casos por departamento, encontramos que las minorías étnicas tenían mayores probabilidades de morir (OR = 2,03; $\mathrm{IC}_{95 \%}$ 1,61- 2,56; $\left.\mathrm{p}<0,001\right)$.

Discusión. Encontramos una asociación entre la pertenencia a una minoría étnica y una mayor probabilidad de mortalidad con lesiones causadas por minas antipersona. Estos hallazgos deberían alentar a los legisladores de las zonas rurales de Colombia a trabajar más diligentemente, para reducir las consecuencias nocivas de las lesiones causadas por estos artefactos en los grupos étnicos minoritarios.

Palabras clave: heridas y traumatismos; traumatismos por explosión; mortalidad; disparidades en el estado de salud; grupos étnicos; Colombia.

Fecha de recibido: 15/09/2020 - Fecha de aceptación: 31/03/2021 - Fecha de publicación en línea: 10-06-2021 Correspondencia: Ramiro Manzano-Núñez, Calle 14aㅡ oeste \#55-175, Cali, Colombia. Teléfono: 310-2710403 Correo electrónico: ramiro.manzano@urosario.edu.co

Citar como: Rodríguez-Narváez JG, Castillo A, González J, González A, Manzano-Núñez R, González F, García AF. Disparidades por etnia en la mortalidad por minas antipersona: Un análisis a nivel país. Rev Colomb Cir. 2021;36:677-81. https://doi.org/10.30944/20117582.829

Este es un artículo de acceso abierto bajo una Licencia Creative Commons - BY-NC-ND https://creativecommons.org/licenses/by-ncnd/4.0/deed.es 


\begin{abstract}
Introduction. The Colombian Victims of Antipersonnel Mines Injuries registry was launched by the Colombian government with the objective of collecting information on all the cases of injuries caused by antipersonnel landmines in the country. The purpose of this study was to investigate the mortality disparities among ethnic minority victims of antipersonnel landmine injuries.
\end{abstract}

Methods. A multivariate logistic regression was performed to examine the association between ethnic minorities and mortality in people injured by antipersonnel mines.

Results. A total of 10,306 cases of injuries caused by antipersonnel landmines were registered, of which 430 were people belonging to minority ethnic groups (indigenous or Afro-descendant). Of these, 85 (19.7\%) were women and $156(36.2 \%)$ were under 18 years of age. Almost all people from ethnic minority groups were located in rural areas $(n=427,99.3 \%)$ and mortality was significantly higher compared to the mestizo population (mestizo 18.5\% vs. individuals from ethnic minorities $29,3 \%$; $<<0.001$ ). After adjusting for sex, age group, active duty soldier status, rural area, and case volume for each geographic department, we found that ethnic minorities were more likely to die after suffering an antipersonnel mine injury (OR $=2.03 ; 95 \%$ CI 1.61-2.56; $\mathrm{p}<0.001)$.

Discussion. We found an association between belonging to an ethnic minority and a higher probability of mortality with injuries caused by antipersonnel mines. These findings should encourage legislators in rural Colombia to work more diligently to reduce the harmful consequences of injuries caused by these devices in ethnic minority groups.

Keywords: wounds and trauma; blast injuries; mortality; disparities in health status; ethnic groups; Colombia.

\section{Introducción}

Colombia sufrió, por décadas, un conflicto armado que dejó miles de víctimas, civiles y militares, y causó una crisis de seguridad de dimensiones catastróficas ${ }^{1,2}$. Durante los últimos 50 años del conflicto hubo aproximadamente 92.426 víctimas de acciones hostiles y, desde 1988, ocurrieron alrededor de 39.000 muertes debidas al conflicto armado ${ }^{3}$.

Desde una perspectiva global, se ha estimado que anualmente unas 800.000 personas son víctimas de heridas de guerra que requieren admisión hospitalaria; de estas, una proporción importante es causada por minas antipersona ${ }^{4}$. En Colombia, las minas antipersona causaron 11.929 víctimas de trauma, lo que puso al país como una de las regiones con la mayor tasa de víctimas por estos artefactos a nivel mundial ${ }^{5}$.

La carga de trauma y violencia generada por los conflictos armados podría ser de mayor magnitud en minorías, individuos con un estado socio-económico de base bajo y poblaciones vulnerables ${ }^{6}$. En el contexto de las minas antipersona, los efectos podrían ser peores ya que estos artefactos se ubican más comúnmente en áreas rurales, en donde las personas pertenecen a estratos socioeconómicos más bajos o a minorías étnicas (indígenas o afrodescendientes).

El propósito de este estudio fue estudiar las disparidades en mortalidad como función de la pertenencia a minorías étnicas entre víctimas de minas antipersona en Colombia.

\section{Métodos}

Este estudio se reportó de acuerdo con la guía STROBE para estudios observacionales.

\section{Fuente de datos}

Para este estudio observacional, utilizamos datos del Registro Colombiano de Víctimas de Lesiones por Minas Antipersonal (base de datos MAP/ MUSE), que está completamente disponible en línea y proporciona información pública, anonimizada, de todas las personas que han sido víctimas de lesiones por minas antipersona en Colombia, desde 1991.

La base de datos MAP/MUSE es un registro patrocinado por el gobierno de la República de 
Colombia, que se lanzó en el año 2001, con el objetivo de recopilar datos de todos los casos de lesiones por minas antipersona en el país. Aunque inicialmente, el registro recopiló datos retrospectivamente de 1991 a 2001, la recolección prospectiva y sistemática de información comenzó a partir del 2002.

\section{Población de estudio}

Los registros de la base de datos MAP/MUSE se extrajeron de los años 2002 a 2018 y se incluyeron todas las personas registradas en la base de datos durante este período. La base de datos contiene información sobre datos demográficos y resultados. Específicamente, los elementos de datos centrales en el MAP/MUSE incluyen edad, género, origen étnico, lugar de la lesión y resultado (vivo o muerto). También contiene datos sobre si la víctima era un soldado o un civil.

\section{Análisis estadístico}

Las diferencias en las características de las víctimas de las minas terrestres se compararon entre individuos de minorías étinicas y la población mestiza, utilizando una prueba de chi-cuadrado para variables categóricas. La mortalidad se calculó dividiendo el número de eventos (muerte) por el número total de víctimas de las minas terrestres antipersonal.

Se utilizó un modelo de regresión multivariable, ajustado por sexo, grupo de edad, raza, ocupación (soldado en servicio vs. civil), área rural y zonas geográficas (departamentos), de acuerdo con el volumen de casos, para estudiar la asociación entre la pertenencia a un grupo étnico y la mortalidad por minas antipersona. Todos los análisis se realizaron en el software Stata ${ }^{\circledR}$, versión 14 (StataCorp. LP, College Station, TX, USA).

\section{Resultados}

Durante el periodo observado se registró un total de 10.306 casos de lesiones por minas antipersona, de los cuales 430 pertenecían a grupos étnicos minoritarios (indígenas o afrodescendientes). La tabla 1 presenta las características generales de la cohorte estudiada.

De todos los individuos víctimas por minas antipersona, 85 (19,7\%) eran mujeres y 156 $(36,2 \%)$ eran menores de 18 años. Casi todas las personas de grupos étnicos minoritarios se encontraban en zonas rurales $(n=427 ; 99,3 \%)$. La mortalidad fue significativamente mayor entre los individuos de grupos étnicos minoritarios, en comparación con la población mestiza (mestizos $18,5 \%$ vs. individuos de minorías étnicas $29,3 \%$; $<<0,001$ ).

Después de ajustar por sexo, grupo de edad, estado de soldado en servicio activo, área rural y volumen de casos por departamentos geográficos, encontramos que las minorías étnicas tenían mayores probabilidades de mortalidad ajustadas al riesgo después de sufrir una lesión por minas terrestres $\left(\mathrm{OR}=2,03 ; \mathrm{IC}_{95 \%} 1,61-2,56\right.$; $\mathrm{p}<0,001$ ).

Tabla 1. Características generales de toda la cohorte de víctimas de minas antipersona. Colombia, 2002-2018.

\begin{tabular}{lcccc}
\hline & Todos $(\mathbf{n = 1 0 . 3 0 5})$ & Minorías $(\mathbf{n = 4 3 0})$ & Mestizos $(\mathbf{n = 9 8 7 5 )}$ & Valor $\mathbf{p}$ \\
\hline Género (Mujer), $\mathrm{n}(\%)$ & $497(4,8 \%)$ & $85(19,7 \%)$ & $412(4,1 \%)$ & $<0,001$ \\
Edad (menores de edad), $\mathrm{n}(\%)$ & $993(9,6 \%)$ & $156(36,3 \%)$ & $837(8,5 \%)$ & $<0,001$ \\
Área rural, $\mathrm{n}(\%)$ & $10.199(98,9 \%)$ & $427(99,3 \%)$ & $9772(98,9 \%)$ & 0,4 \\
Ocupación & & & & $<0,001$ \\
Civiles, $\mathrm{n}(\%)$ & $3980(38,6 \%)$ & $430(100 \%)$ & $3550(36 \%)$ & \\
Soldados, $\mathrm{n}(\%)$ & $0325(61,3 \%)$ & $0(0 \%)$ & $6325(64 \%)$ & \\
Mortalidad, $\mathrm{n}(\%)$ & $1961(19 \%)$ & $126(29,3 \%)$ & $1835(18,5 \%)$ & $<0,001$ \\
\hline
\end{tabular}




\section{Discusión}

En este estudio usamos datos de más de diez mil víctimas de minas antipersona, recopilados por un periodo de 16 años, y encontramos que la probabilidad ajustada de muerte luego de sufrir una lesión con este tipo de artefactos fue significativamente mayor en individuos pertenecientes a grupos étnicos minoritarios, específicamente en comunidades negras e indígenas. Estos resultados son preocupantes y deberían impulsar la creación e implementación de políticas públicas encaminadas a disminuir el impacto de esta disparidad, sobretodo en áreas rurales de Colombia.

El principal hallazgo de este estudio fue que el riesgo ajustado de morir fue mayor para individuos de grupos étnicos catalogados como minorías. Estudios previos han mostrado que los grupos raciales entendidos como minorías se han visto afectados en mayor magnitud luego de sufrir trauma ${ }^{7-9}$. Evidencia de otras latitudes muestra que los pacientes de raza negra tienen una probabilidad mayor de morir en el contexto de traumatismos al ser comparados con pacientes de raza blanca. Nuestro estudio muestra resultados similares, siendo el mecanismo de trauma las minas antipersonas.

En Colombia este fenómeno ha persistido durante los últimos 50 años, desencadenado por el conflicto armado, y es nuestra intención poner en evidencia que las poblaciones minoritarias son las que pueden sufrir peores desenlaces. Estos resultados podrían servir para procurar reducir las disparidades que existen en la atención en salud. Algunas intervenciones que pensamos podrían reducir las diferencias entre poblaciones son mejorar la cobertura del sistema de salud y generar programas de prevención dirigidos a poblaciones más vulnerables, las cuales durante años se han visto marginadas en nuestro país.

Aunque encontramos una disparidad en la mortalidad como función de la pertenencia a un grupo étnico, es necesario mencionar que este hallazgo podría deberse a otros factores. Por ejemplo, nuestros resultados podrían estar permeados por factores de confusión, dado que las comunidades minoritarias tienden a vivir en regiones alejadas, donde el acceso a servicios de salud es limitado. En este escenario, no sería la pertenencia a un grupo étnico lo que estaría condicionando una mayor mortalidad, sino la deficiencia en el acceso a servicios de salud, sobretodo pre-hospitalarios, lo que podría relacionarse con una menor probabilidad de supervivencia. Sin embargo, nuestros resultados son plausibles y lógicos a la luz del contexto colombiano, y lo que se sabe hasta ahora sobre las disparidades raciales en trauma, que concuerdan con hallazgos similares en otras poblaciones y otras latitudes ${ }^{6}$.

Nuestro estudio tiene limitaciones y los resultados deben interpretarse a la luz de los análisis realizados y la base de datos utilizada. La principal limitación de este estudio es la falta de información sobre otras variables, que podría haber mejorado el ajuste en el análisis multivariado. Al usar una base de datos administrativa, como la base MAP/MUSE, hay un déficit de la granularidad de la información o nivel de detalle de los datos. Es decir, hay ausencia de variables pre-hospitalarias y clínicas que se asocian a la probabilidad de morir, pero que no podemos incluir en nuestro modelo multivariado.

\section{Conclusión}

Este estudio encontró una asociación entre pertenecer a un grupo étnico minoritario y tener mayor probabilidad ajustada de muerte luego de sufrir un trauma por una mina antipersona. Estos hallazgos son preocupantes y merecen más estudios y una mayor atención por parte del gremio médico y los tomadores de decisiones en Colombia.

\section{Consideraciones éticas}

Consentimiento informado: La información utilizada para este estudio se considera un conjunto de datos de uso público, preparado con la intención de ponerlo a disposición de terceros. Los datos disponibles para el público no son identificables individualmente $y$, por lo tanto, su análisis no involucraría a sujetos humanos. Por lo tanto, se consideró exento de la revisión del comité de ética, siguiendo las recomendaciones proporcionadas por la guía creada por el comité de ética de la Universidad del Rosario.

Conflicto de interés: Ninguno declarado por los autores.

Financiación: Autofinanciada por los autores. 


\section{Contribuciones de los autores:}

- Concepción y diseño del estudio: José G. RodríguezNarváez, Andrea Castillo, Jessica González, Alejandro González.

- Adquisición de datos: los datos fueron obtenidos de una fuente de datos pública adscrita al gobierno nacional de Colombia. Pueden ser encontrados en el sitio web Accion Contra Minas http://www.accioncontraminas. gov.co/ Ramiro Manzano-Núñez fue el encargado de obtener la base de datos.

- Análisis e interpretación de datos: Alejandro González, Felipe González, Ramiro Manzano-Núñez.

- Redacción del manuscrito: José G. Rodríguez-Narváez, Andrea Castillo, Jessica González, Alejandro González.

- Revisión crítica: José G. Rodríguez-Narváez, Andrea Castillo, Jessica González, Alberto F. García, Felipe González, Ramiro Manzano-Núñez.

\section{Referencias}

1. Ordoñez CA, Manzano-Nunez R, Naranjo MP, Foianini E, Cevallos C, Londoño MA, et al. Casualties of peace: an analysis of casualties admitted to the intensive care unit during the negotiation of the comprehensive Colombian process of peace. World J Emerg Surg. 2018;13:2. https://doi.org/10.1186/s13017-017-0161-2

2. Ordoñez CA, Manzano-Nunez R, Parra MW, Herrera JP, Naranjo MP, Escobar SS, et al. Analysis of combat casualties admitted to the emergency department during the negotiation of the comprehensive Colombian process of peace. Colomb Med. 2017;48:155-60. https://doi.org/10.25100/cm.v43i4.3389
3. Rubiano AM, Sánchez ÁI, Guyette F, Puyana JC. Trauma care training for National Police nurses in Colombia. Prehosp Emerg Care. 2010;14:124-30. https://doi.org/10.3109/10903120903349762

4. Haagsma JA, Graetz N, Bolliger I, Naghavi M, Higashi $\mathrm{H}$, Mullany EC, et al. The global burden of injury: incidence, mortality, disability-adjusted life years and time trends from the Global Burden of Disease study 2013. Inj Prev. 2015;22:3-18. https://doi.org/10.1136/injuryprev-2015-041616

5. Daniels JP. Helping Colombia's landmine survivors. Lancet. 2016;387:2079-80. https://doi.org/10.1016/S0140-6736(16)30597-9

6. Haider AH, Weygandt PL, Bentley JM, Monn MF, Rehman KA, Zarzaur BL, et al. Disparities in trauma care and outcomes in the United States: A systematic review and meta-analysis. J Trauma Acute Care Surg. 2013;74:1195-205. https://doi.org/10.1097/01586154-201305000-00002

7. Crompton JG, Pollack KM, Oyetunji T, Chang DC, Efron DT, Haut ER, et al. Racial disparities in motorcycle-related mortality: an analysis of the National Trauma Data Bank. Am J Surg. 2010;200:191-6. https://doi.org/10.1016/j.amjsurg.2009.07.047

8. Shafi S, Marquez de la Plata C, Diaz-Arrastia R, Shipman $\mathrm{K}$, Carlile M, Frankel H, et al. Racial disparities in longterm functional outcome after traumatic brain injury. J Trauma Acute Care Surg. 2007;63:1263-70. https://doi.org/10.1097/TA.0b013e31815b8f00

9. Glance LG, Osler TM, Mukamel DB, Meredith JW, Li Y, Qian F, Dick AW. Trends in racial disparities for injured patients admitted to trauma centers. Health Serv Res. 2013;48:1684-703. https://doi.org/10.1111/1475-6773.12064 